Encontros Bibli: revista eletrônica de biblioteconomia e ciência da informação, $v .17, n$. esp. 2 - III SBCC, p.227-237, 2012. ISSN 1518-2924. DOI:10.5007/15182924.2012v17nesp $2 p 227$

\title{
SEER E PDF/A NA PRESERVAÇÃO DO PERIÓDICO ARCHIVOS RIO- GRANDENSES DE MEDICINA: RELATO DE EXPERIÊNCIA
}

\author{
Ana Paula Araújo Cabral da Silvai ${ }^{\mathrm{i}}$
}

\begin{abstract}
Resumo: $O$ artigo narra o processo de digitalização de um periódico do acervo histórico, da Biblioteca FAMED/HCPA (Faculdade de Medicina da Universidade Federal do Rio Grande do Sul - UFRGS e Hospital de Clínicas de Porto Alegre), chamado "Archivos Rio-grandenses de Medicina". Este relato inclui breve revisão teórica acerca das mudanças no processo de editoração e divulgação das publicações periódicas científicas, bem como do desenvolvimento das tecnologias de preservação e acesso. Também descreve a conversão dos representantes digitais dos artigos para o formato PDF/A e a disponibilização online, por meio do Portal SEER UFRGS, dos fascículos da revista. Por fim, destaca a importância dos metadados na indexação e recuperação da informação.
\end{abstract}

Palavras-chave: Revista científica. Preservação digital. Indexação. Metadado.

\section{SEER AND PDF/A IN PRESERVATION OF ARCHIVOS RIO-GRANDENSES DE MEDICINA: EXPERIENCE REPORT}

\begin{abstract}
The article describes the process of scanning a journal of the historical collection, from the Biblioteca FAMED/HCPA (Faculdade de Medicina da Universidade Federal do Rio Grande do Sul - UFRGS e Hospital de Clinicas de Porto Alegre), called "Archivos Rio-grandenses de Medicina". This report includes a brief theoretical review about the changes in the process of publishing and dissemination of scientific journals, as well as the development of technologies for preservation and access. It also describes the conversion of digital representatives of the articles to PDF/ and available online through the Portal SEER UFRGS, of the issues of the journal. Finally, it highlights the importance of metadata for indexing and information retrieval.
\end{abstract}

Keywords: Scientific journals. Digital preservation. Indexing. Metadata.

\footnotetext{
${ }^{i}$ Biblioteca FAMED/HCPA (Faculdade de Medicina - UFRGS/Hospital de Clínicas de Porto Alegre) ana.cabral@ufrgs.br
} 


\section{INTRODUÇÃO}

A Biblioteca FAMED/HCPA possui em seu acervo diversos periódicos da área médica, editados no Rio Grande do Sul, como por exemplo: "Revista dos Cursos", "Revista Científica CASL (Centro Acadêmico Sarmento Leite)", "Anais da Faculdade de Medicina de Porto Alegre", entre outros títulos.

Nesta categoria de publicação, destaca-se “Archivos Rio-Grandenses de Medicina”, o qual era um órgão de divulgação da Sociedade de Medicina de Porto Alegre. Começou a ser publicado em janeiro de 1920, foi encerrado em junho de 1943 e sua coleção impressa completa encontra-se armazenada no acervo histórico da Biblioteca FAMED/HCPA.

Por causa das características peculiares do suporte físico dos fascículos e, consequentemente, do desgaste natural pelo manuseio, os exemplares estão disponíveis somente para consulta local, com permissão para serem fotocopiados.

No entanto, devido a sua importância histórica e buscando manter o propósito original de sua publicação, ocorreu a decisão de se buscarem ferramentas para preservar o seu conteúdo informacional e também ampliar a divulgação dessa produção intelectual.

\section{REVISÃO DE LITERATURA}

Desde os seus primórdios, até os anos noventa do século $\mathrm{XX}$, as coleções de periódicos científicos existentes nas bibliotecas universitárias brasileiras, eram predominantemente em formato impresso (STUMPF, 1996). Neste contexto, a preocupação com a manutenção destes acervos envolvia estudos sobre as características deste tipo de material e a proposição de ações para a sua conservação.

Dentre as opções existentes, a microfilmagem e o posterior arquivamento em condições ambientais adequadas são considerados por diversos autores uma solução satisfatória no que tange à expectativa de durabilidade do microfilme e na confiabilidade do processo (ELKINGTON, 2001; FOX, 2001; JONES, 2001; MARDERO ARELLANO, 2008; OGDEN, 2001; VAN BOGART, 1995, 1997; WATERS, 2001).

No entanto, os autores acima citados reconhecem que esta alternativa apresenta limitações nos recursos humanos, financeiros e de acessibilidade. Neste sentido, ELKINGTON (2001, p.16) destaca que a microfilmagem “'[...] é uma opção cara e 
tecnicamente complexa, que deve ser realizada com pleno conhecimento dos custos e benefícios $[\ldots] "$.

Além disso, o advento de novas mídias eletrônicas (Compact Disc - CD, Digital Versatile Disk - DVD, etc.) e o desenvolvimento das tecnologias de informação e comunicação (internet, digitalização, OCR - Reconhecimento Óptico de Caracteres, etc.), fez surgirem outros questionamentos e também outras possibilidades, envolvendo tanto a geração quanto a preservação das publicações periódicas científicas.

No que diz respeito às indagações, estas se referem basicamente: à definição das condições físicas e lógicas para armazenamento dos representantes digitais ${ }^{2}$ e também dos documentos nato-digitais ${ }^{3}$; ao inevitável e progressivo processo de obsolescência de dispositivos tecnológicos (hardware) e programas de computadores (software); aos custos intrínsecos envolvidos - operacionais, humanos e financeiros - nos projetos de preservação (FERREIRA, 2006; MÁRDERO ARELLANO, 2008; WESING, 2010).

Como respostas a estes questionamentos, surgiram diversas iniciativas, como a criação de repositórios digitais para arquivamento de periódicos eletrônicos. Neste aspecto, destacamse o JSTOR (Journal Storage: The Scholarly Journal Archive), um consórcio americano, cuja proposta é armazenar e disponibilizar, mediante pagamento, coleções eletrônicas retrospectivas. Outro projeto semelhante é o LOCKSS (Lots of Copies keeps Staff Save), um software livre, que requer um menor investimento em equipamentos e uma administração técnica mínima. Sobre ele, Márdero Arellano (2008, p. 115) afirma:

Considerando o alto custo do armazenamento em repositórios digitais, uma metodologia como a do LOCKSS, é muito importante para países em desenvolvimento, onde a maioria das instituições carece de financiamento para as atividades relacionadas com a preservação digital de publicações científicas.

Outras proposições a serem destacadas, na gestão (geração/preservação) dos periódicos eletrônicos são: o Projeto SciELO (Scientific Electronic Library), desenvolvido pela Fundação de Apoio a Pesquisa do Estado de São Paulo (Fapesp) em parceria com o Centro Latino-Americano e do Caribe de Informação em Ciências da Saúde da Organização Pan-Americana da Saúde (BIREME/OPAS/OMS); o SEER (Sistema Eletrônico de Editoração de Revistas), tradução e customização do OJS (Open Journal System), feita pelo IBICT.

\footnotetext{
${ }^{2}$ Representações em formato de arquivo digital de um documento originalmente não digital (CONSELHO NACIONAL DE ARQUIVOS, 2010)

${ }^{3}$ Documentos que já nascem no formato digital (DZIEKANIAK, 2010) 
Meirelles (2009, p. 42), afirma que os sistemas de Modelo Acesso Aberto ${ }^{4}$, como é o caso do SciELO e do SEER, são mais vantajosos, por "[...] possibilitar a integração e descentralização do processo de produção do conhecimento, o que garante a preservação digital dos originais [...]".

A recomendação do $\mathrm{PDF} / \mathrm{A}$, como o formato de preservação a longo prazo de documentos eletrônicos, também pode ser considerada uma importante iniciativa. A norma, homologada pela ISO (International Organization for Standardization) em 2005, foi traduzida e disponibilizada no Brasil em 2009, com o nome de "Gerenciamento de documentos Formato eletrônico de arquivo de documento para preservação de longo prazo Parte 1: Uso do PDF 1.4 (PDF/A-1)".

No Brasil, o Conselho Nacional de Arquivos (CONARQ) também recomenda o uso deste formato digital, por suas características de acessibilidade e fidedignidade ao original, “[...] especialmente quando esse é formado por múltiplas páginas e contiver também imagens fixas.” Sobre este assunto, WENSING (2010, p. 47) enfatiza: “As especificações produzidas e mantidas por órgãos normatizados são mais seguras e tem mais garantia de disponibilidade por longo prazo, sendo assim são os mais recomendados para preservação digital”.

Outra proposição relevante foi a descrição em metadados, considerados por diversos autores, estratégias válidas para a autenticidade dos dados e a integridade do conteúdo (BORBA, 2009; MÁRDERO ARELLANO, 2008; MEIRELLES, 2009; WESING, 2010). Dentre as iniciativas, destaca-se a pioneira OAI (Open Archives Initiative).

De acordo com Baptista et al. (2007, p.10) este modelo “[...] proporciona alto nível de interoperabilidade, por meio de dois grandes pilares: 1) padrão de metadados Dublin Core; 2) protocolo de coleta de metadados, denominado OAI-PMH."

Embora as propostas descritas nos parágrafos precedentes não sejam uma unanimidade na literatura (BORBA, 2009; FOX, 2001; JONES, 2001; MÁRDERO ARELLANO, 2008; MEIRELLES, 2009; OGDEN, 2001; VAN BOGART, 1997; WATERS, 2001; WESING, 2010), elas estão entre as que melhor respondem aos questionamentos anteriormente citados. A razão dessa aceitação reside no fato das mesmas estarem baseadas em projetos de Modelo Acesso Aberto e/ou por seguirem as recomendações de instituições normatizadoras, como a ISO.

\footnotetext{
${ }^{4}$ O Modelo Acesso Aberto é baseado no Movimento de Acesso livre à Informação Científica [...] um movimento mundial, baseado em uma série de manifestos e conferências, que propõe a utilização desse modelo em prol do acesso livre à informação científica. Para sua operacionalização é utilizado o modelo tecnológico desenvolvido pela OAI, que serve para operacionalizar o intercâmbio de dados entre sistemas, através do protocolo OAIPMH. (MEIRELLES, 2009, p. 59). 230
} 
Cabe ressaltar ainda que vários autores (BAPTISTA et al., 2007; BORBA, 2009; MÁRDERO ARELLANO, 2008; MEIRELLES, 2009) consideram o estabelecimento de políticas bem definidas, tanto em instituições governamentais quanto acadêmicas, como um fator imprescindível no êxito de um projeto de preservação digital.

\section{RELATO DE EXPERIÊNCIA}

O projeto de preservação digital e divulgação on-line dos "Archivos Rio-grandenses de Medicina" iniciou em abril de 2011. A primeira etapa foi a definição do repositório para armazenamento/difusão da coleção, na qual consideraram-se apenas aqueles existentes na UFRGS: o Lume (http://www.lume.ufrgs.br) e o Portal SEER UFRGS (http://seer.ufrgs.br). Essa decisão foi tomada visando otimização dos recursos (financeiros, humanos e operacionais), bem como aproveitamento do conhecimento técnico já adquirido na instituição, no manejo dessas ferramentas.

Avaliando os dois portais, perceberam-se características comuns, como compatibilidade com o Protocolo de Arquivos Abertos (OAI), possibilidade de arquivamento dos documentos em formato PDF e uso do padrão Dublin Core para metadados. No entanto, embora o Lume inclua em seu acervo artigos de periódicos, e contemple em seus objetivos ${ }^{5}$ a inclusão de publicações como os "Archivos Rio-Grandenses de Medicina", determinadas funcionalidades do SEER foram determinantes na escolha deste como repositório da revista, como:

- organização da revista por volumes e fascículos (http://seer.ufrgs.br/riograndemed/issue/archive), facilitando a visualização da coleção;

- recuperação dos artigos por edição, autor, título, resumo, documento(s) suplementar(es), termos indexados (área do conhecimento, assunto, tipo (método/foco), cobertura) e texto completo, ampliando as possibilidades de divulgação do conteúdo;

- arquivamento dos documentos em formato PDF/A e no sistema LOCKSS, otimizando a preservação digital da revista;

\footnotetext{
${ }^{5}$ V- Promover o acesso a outros documentos de relevância para a Instituição, que fazem parte de seus acervos, embora não produzidos por ela, maximizando o seu uso [...] (UNIVERSIDADE FEDERAL DO RIO GRANDE DO SUL, 2010, p. 2)
} 
- customização do SEER, ajustando-o às características dos “Archivos Rio-Grandenses de Medicina", no que tange à periodicidade (publicação encerrada) e formatação (mudanças frequentes na apresentação/disposição das seções nos fascículos).

Após a definição do Portal SEER UFRGS como repositório, seguiu-se um período de capacitação no uso do SEER, mediante treinamento promovido pela Pró-Reitoria de Pesquisa (PROPESQ), na UFRGS. Com essa instrumentalização básica, passou-se à customização do sistema, definindo-se primeiramente o Editor Gerente e, consequentemente, os ajustes da revista, por meio das Páginas de Gerenciamento (http://seer.ufrgs.br/riograndemed/help/view/journal/topic/000001).

Concomitante a este processo decisório e de implementação, ocorreu a digitalização da totalidade dos fascículos (realizada por um graduando do curso de História), para o qual foram utilizados os equipamentos e programas listados a seguir. A escolha destes foi determinada por uma busca na redução dos custos na execução do projeto e um uso proveitoso dos subsídios já existentes:

- Multifuncional Toshiba, modelo e-STUDIO 282/283, a qual utiliza o software Toshiba e-STUDIO File Downloader, versão 3.1.11.0 - empregada para escanear e digitalizar os originais;

- Microcomputadores, com Sistemas Operacionais Windows XP e 7 Professional, usados respectivamente na conversão dos fascículos e na inserção destes no Portal SEER UFRGS;

- Adobe Acrobat 8 Professional, versão 8.1.4 e Adobe Acrobat X Pro - utilizados para o tratamento físico da obra digitalizada (extração de manchas e sombras das páginas, OCR), bem como a conversão para o formato PDF/A-1.

Nesta etapa, no aspecto preservação, três decisões devem ser destacadas. A primeira diz respeito à escolha do PDF/A-1, como padrão de arquivamento dos representantes digitais dos artigos dos "Archivos Rio-grandenses de Medicina". Essa escolha deve-se tanto por ser um formato recomendado por uma instituição referencial (ISO), mas igualmente devido as suas características. Por ser autodescritivo, implica na existência de metadados XMP, imprescindíveis na conservação das informações acerca do arquivo, bem como nas ferramentas de busca.

Porém, apesar de todos os recursos utilizados, determinadas dificuldades surgiram no 
processo de digitalização. As principais problemáticas se referiram às condições dos originais dos fascículos dos “Archivos Rio-Grandenses de Medicina”. Por serem um material impresso em papel, um suporte suscetível à instabilidade química, determinados exemplares sofreram degradação física, demonstrada através de manchas, folhas quebradiças e também do desbotamento da impressão. Além disso, por ter sido elaborada no início do século $\mathrm{XX}$, o projeto gráfico da revista utiliza fontes de letras que dificultam a identificação das palavras. Com isso, determinadas páginas requereram maior atenção no processo de conversão para o formato digital, para evitar a perda de informações relevantes. Ainda assim, mesmo com o uso do OCR, certas palavras não podem ser localizadas pela ferramenta de pesquisa no PDF.

Após este processo de conversão do formato impresso para o digital, partiu-se para a segunda decisão, a feitura de uma cópia de segurança da coleção digitalizada. Selecionou-se o suporte DVD, o qual será arquivado juntamente com os demais exemplares da seção digital, do acervo da Biblioteca FAMED/HCPA. Esta providência objetiva tornar mais abrangente e eficaz a conservação dos representantes digitais do acervo impresso

A última definição, neste contexto de preservação, mencionada na seção "Sobre" da revista, foi a de que os artigos apresentados na versão eletrônica seguirão a formatação utilizada nas edições originais, inclusive quanto à grafia da Língua Portuguesa. Esta determinação, bem como a manutenção da organização interna (paginação, disposição dos textos/imagens) foi tomada visando a salvaguarda destes aspectos históricos do periódico.

A terceira fase do projeto é a inclusão dos fascículos no Portal SEER UFRGS, a qual será progressiva, até que o conteúdo de todos os fascículos esteja acessível. Atualmente, encontram-se disponibilizados no Portal do SEER UFRGS os volumes relativos aos anos de 1920 a 1929 (http://seer.ufrgs.br/riograndemed/issue/archive).

Visando agilizar a inserção dos artigos e também por inexistir a avaliação por pares, foram necessárias adequações no processo de submissão. Para tanto, o profissional responsável pela inclusão assumiu os papéis de Editor-Gerente, Editor, Editor de Seção e Autor. Estes ajustes resultaram na redução das etapas, pois o arquivo submetido pode ser encaminhado diretamente para o último passo da Edição (composição final e agendamento para publicação).

Ainda cabe destacar que a inserção no Portal SEER UFRGS pressupõe um trabalho minucioso de leitura e indexação de cada artigo. Como a revista foi escrita nas décadas de 1920/1940 do século XX, a grafia pode requerer uma interpretação mais apurada por parte do indexador. Outro detalhe a salientar, é o fato de a revista ter sido publicada por uma instituição vinculada à área da saúde. Essa característica levou à opção pelo uso dos termos do 
vocabulário estruturado DeCS (Descritores em Ciências da Saúde), na definição das palavraschave, nos metadados de submissão. Esta medida visa proporcionar tanto a consistência na pesquisa quanto valer-se da já existente familiaridade dos usuários com a terminologia, ampliando a divulgação e a revocação da revista.

\section{CONSIDERAÇÕES FINAIS}

O projeto encontra-se em implementação, sendo necessários, portanto, ajustes e adequações, tanto do periódico ao software, quanto ao conhecimento e manejo das funcionalidades do SEER, em suas novas versões. No entanto, por utilizar o software SEER, que apresenta o sistema LOCKSS para arquivamento e o OAI-PMH como protocolo de coleta de metadados, os objetivos de preservação do conteúdo, bem como da ampliação da divulgação da revista, podem ser satisfatoriamente alcançados.

Além disso, o uso do PDF/A-1 como um formato de arquivamento, embora tenha sido recomendado pela ISO e utilizado por diversas instituições, ainda está em observação pelos pesquisadores. Por isso, optou-se por conservar os originais em papel, no acervo histórico, aguardando a avaliação do processo de digitalização, nos quesitos autenticidade do documento digitalizado, segurança do repositório externo (LOCKSS) e disponibilidade.

Outrossim, após a finalização da inserção dos fascículos faz-se pertinente um estudo sobre a acessibilidade e usabilidade da coleção, disponibilizada no formato digital e on-line. Essa iniciativa é relevante, visto que existem outras obras do acervo na Biblioteca FAMED/HCPA, com características (local de publicação, condições do suporte, importância histórica do conteúdo para a instituição) semelhantes aos "Archivos".

Ainda com relação ao SEER, destaca-se que, embora tenha sido desenvolvido para uso com periódicos correntes, a possibilidade de customização do software foi facilmente aplicável (sem a necessidade de auxílio de pessoal especializado, da área de informática) e contemplou as demandas/peculiaridades de uma revista de publicação encerrada,. A experiência e o conhecimento adquiridos com este processo certamente poderão ser empregados em projetos vindouros. 


\section{REFERÊNCIAS}

BORBA, Vildeane da Rocha. Modelo orientador para construção de estratégias de preservação digital: estudo de caso do Banco de Teses e Dissertações da UFPE. 2009. 133 f. Dissertação (Mestrado em Ciência da Informação)-Universidade Federal da Paraíba, João Pessoa, 2009. Disponível em: $<$ $<$ http://dci2.ccsa.ufpb.br:8080/jspui/bitstream/123456789/165/1/Dissertacao_VILDEANE_PP GCI_UFPB.pdf $>$. Acesso em: 27 mar. 2012.

CONSELHO NACIONAL DE ARQUIVOS. Recomendações para digitalização de documentos arquivísticos permanentes. Brasília: 2010. Disponível em: $<$ http://www.conarq.arquivonacional.gov.br/media/publicacoes/recomenda/recomendaes_para _digitalizao.pdf>. Acesso em: 21 mar. 2012.

CONWAY, Paul. Preservação no universo digital. 2. ed. Rio de Janeiro: Projeto Conservação Preventiva em Bibliotecas e Arquivos; Arquivo Nacional, 2001. 32 p. (Projeto de Conservação Preventiva em Bibliotecas e Arquivos, 52). Disponível em: $<$ http://www.prefeitura.sp.gov.br/cidade/upload/cpba_52_1253284406.pdf $>$. Acesso em: 19 mar. 2012.

DZIEKANIAK, Gisele. A organização da informação e a comunicação científica: implicações para os profisssionais e usuários da informação. Em Questão, Porto Alegre, v. 16, n. 1, p. 45-59, jan./jun. 2010. Disponível em:

$<$ http://seer.ufrgs.br/EmQuestao/article/view/11434>. Acesso em: 21 mar. 2012.

ELKINGTON, Nancy (Ed.). Manual do RLG para microfilmagem de arquivos. 2. ed. Rio de Janeiro: Projeto Conservação Preventiva em Bibliotecas e Arquivos; Arquivo Nacional, 2001. 205 p. (Projeto de Conservação Preventiva em Bibliotecas e Arquivos, 53). Disponível em: <http://www.prefeitura.sp.gov.br/cidade/upload/cpba_53_1253284434.pdf>. Acesso em: 27 mar. 2012.

FERREIRA, Miguel. Introdução à preservação digital: conceitos, estratégias e actuais consensos. Guimarães, Portugal: Escola de Engenharia da Universidade do Minho, 2006. 88 p. Disponível em:

$<$ http://repositorium.sdum.uminho.pt/bitstream/1822/5820/1/livro.pdf $>$. Acesso em: 27 mar. 2012.

FOX, Lisa (Ed.). Microfilmagem de preservação: uma visão geral das decisões administrativas. 2. ed. Rio de Janeiro: Projeto Conservação Preventiva em Bibliotecas e Arquivos; Arquivo Nacional, 2001. 54 p. (Projeto de Conservação Preventiva em Bibliotecas e Arquivos, 48). Disponível em:

$<$ http://www.prefeitura.sp.gov.br/cidade/upload/cpba_48_1253284205.pdf $>$. Acesso em: 27 mar. 2012.

JONES, Lee C. Microfilme para preservação: plataforma para sistemas digitais de acesso. In: OGDEN, Sherelyn et al. Reformatação. 2. ed. Rio de Janeiro: Projeto Conservação Preventiva em Bibliotecas e Arquivos; Arquivo Nacional, 2001. p. 7-10 (Projeto de 
Conservação Preventiva em Bibliotecas e Arquivos, 44-47). Disponível em:

$<$ http://www.prefeitura.sp.gov.br/cidade/upload/cpba_44_a_47_1253284139.pdf $>$. Acesso em: 19 mar. 2012.

MÁRDERO ARELLANO, Miguel Ángel. Critérios para a preservação digital da informação científica. 2008. 354 f. Tese (Doutorado em Ciência da Informação)Universidade de Brasília, Brasília, 2008. Disponível em:

$<$ http://eprints.rclis.org/bitstream/10760/12649/1/Tese_Miguel_\%c3\%81ngel_M\%c3\%a1rder o_Arellano.pdf>. Acesso em: 27 mar. 2012.

MEIRELLES, Rodrigo. Gestão do processo editorial eletrônico baseado no modelo acesso aberto: estudo em periódicos científicos da Universidade Federal da Bahia - UFBA. 2009. 131 f. Dissertação (Mestrado em Ciência da Informação)- Universidade Federal da Bahia, Salvador, 2009. Disponível em: <

$<$ http://eprints.rclis.org/bitstream/10760/14303/1/Disserta\%C3\%A7\%C3\%A3o_Rodrigo_M eirelles_Revisada.pdf $>$. Acesso em: 27 mar. 2012.

OGDEN, Sherelyn. O básico sobre o processo de digitalizar imagens. In: OGDEN, Sherelyn et al. Reformatação. 2. ed. Rio de Janeiro: Projeto Conservação Preventiva em Bibliotecas e Arquivos; Arquivo Nacional, 2001. p. 7-10 (Projeto de Conservação Preventiva em Bibliotecas e Arquivos, 44-47). Disponível em:

$<$ http://www.prefeitura.sp.gov.br/cidade/upload/cpba_44_a_47_1253284139.pdf $>$. Acesso em: 19 mar. 2012.

STUMPF, Ida Regina Chitto. Passado e futuro das revistas científicas. Ciência da Informação, Brasília, DF, v. 25, n.3, 1996. Disponível em:

$<$ http://revista.ibict.br/cienciadainformacao/index.php/ciinf/article/view/463/422>. Acesso em: 19 mar. 2012.

UNIVERSIDADE FEDERAL DO RIO GRANDE DO SUL. Gabinete do Reitor. Portaria n. 5068, de 13 de outubro de 2010. Estabelece a política institucional de informação para o Lume. Disponível em: < http://www.lume.ufrgs.br/arquivos_download/Portaria-5068.pdf $>$. Acesso em: 26 maio 2012.

VAN BOGART, John W. C. Magnetic tape storage and handling: a guide for libraries and archives. Washington, DC: The Comission on Preservation and Acess; St. Paul, MN: National Media Laboratory, 1995. 42 p. Disponível em: <

http://www.eric.ed.gov/PDFS/ED384356.pdf>. Acesso em: 19 mar. 2012.

VAN BOGART, John W. C. Long-Term Preservation of Digital Materials. In: NATIONAL PRESERVATION OFFICE. Preservation and digitisation: principles, practices and policies. London: The National Preservation Office; The British Library, 1997. p. 17-36. Disponível em: <http://www.bl.uk/blpac/pdf/conf1996.pdf >. Acesso em: 19 mar. 2012.

WATERS, Donald J. Do microfilme à imagem digital. 2. ed. Rio de Janeiro: Projeto Conservação Preventiva em Bibliotecas e Arquivos; Arquivo Nacional, 2001. 32 p. (Projeto de Conservação Preventiva em Bibliotecas e Arquivos, 49). Disponível em:

$<$ http://www.prefeitura.sp.gov.br/cidade/upload/cpba_49_1253284217.pdf $>$. Acesso em: 19 mar. 2012. 
WENSING, Jairo. Preservação e recuperação de informação em fontes de informações digitais: estudo de caso do Greenstone. 2010. 219 f. Dissertação (Mestrado em Ciência da Informação)-Universidade Federal de Santa Catarina, Florianopólis, 2010. Disponível em: $<$ http://pgcin.paginas.ufsc.br/files/2010/10/WENSING-Jairo.pdf $>$. Acesso em: 27 mar. 2012. 